\title{
New aggregation operators on group-based generalized intuitionistic fuzzy soft sets
}

\author{
Khizar Hayat ${ }^{1}\left(\mathbb{D} \cdot\right.$ Zalishta Tariq $^{2} \cdot$ Edwin $^{\text {Lughofer }^{3} \cdot \text { M. Fahim Aslam }}{ }^{1}$ \\ Accepted: 20 August 2021 / Published online: 13 September 2021 \\ (c) The Author(s), under exclusive licence to Springer-Verlag GmbH Germany, part of Springer Nature 2021
}

\begin{abstract}
The approach of dealing with uncertainty is enhancing day-by-day with new rudiments and tools which possess their specific qualities. Usually, aggregation operators can easily manage the information in an exact manner. But each operator has different specifications in each problem. In recent few years, aggregation operators on intuitionistic fuzzy soft sets (IFSSs) or generalized intuitionistic fuzzy soft sets (GIFSSs) have been investigated but a lot of improvement is needed to obtain more accurate results. In this research, we defined new aggregation operators on GIFSSs which are used to aggregate our multi-criteria decision-making method. Reasonably, we assigned the weights with both intuitionistic fuzzy values of IFSS and intuitionistic fuzzy values of extra input in a GIFSS, and then by establishing new aggregation operators we appraised the computation in a more precise way. We defined the necessary properties of new aggregation operators and preparatory work of decision making in an algorithm. Then we expressed a real-life application by dint of the proposed methodology. Finally, we presented the comparisons of our work with already existing methods and techniques comprising aggregation operators.
\end{abstract}

Keywords Fuzzy sets · IFSSs · GIFSSs · Aggregation operators for GIFSSs · Multi attributes decision making

\section{Introduction}

\subsection{Background}

In the middle of the twentieth century, mathematician Lotfi Aliasker Zadeh finds out a way to represent the uncertainty of a standard set by fuzzy subset notation (Zadeh 1965). The ordinary sets are not good enough in depicting routine life matters, as they are not able to express any vagueness and

Khizar Hayat

khizar233@gmail.com; khizarhayat@uokajk.edu.pk

Zalishta Tariq

ztariqmscmath@gmail.com

Edwin Lughofer

Edwin.Lughofer@jku.at

M. Fahim Aslam

iamfahimaslam@uokajk.edu.pk

1 Department of Mathematics, University of Kotli, Azad Jammu and Kashmir, Pakistan

2 Department of Mathematics, Women University of Azad Jammu and Kashmir, Bagh, Pakistan

3 Department of Knowledge-Based Mathematical Systems, Johannes Kepler University Linz, Linz, Austria uncertain knowledge of human beings. Conclusively the theory of fuzzy sets was build up with significant applicability in a diverse problem of routine life. This theory is derived on principles from the interval the $[0,1]$, where an item may belong to a set with a certain membership degree. Thus, compared to ordinary sets, it has a better tendency to compel vague information (Zimmermann 2010). Fuzzy sets made many advancements and provide dynamic features in diverse fields such as machine learning, facial pattern recognition, air conditioners, washing machines, transmission systems, control of subway systems, knowledge-based systems for multi-dimensional optimization of power systems, weather forecasting assessments, depicting methods for new product pricing or project risk evaluations, medical diagnosis and treatment plans.

A parameterized generalization of fuzzy sets known as soft sets is built upon a set-valued approximation map to model uncertain concepts by collectively considering various visible features indicated by parameters (Maji et al. 2001a; Molodtsov 1999). In the past ten years, multifarious applications of soft sets have been found out such as in algebraic systems (Acar et al. 2010; Shabir and Naz 2011), engineering designs (Hayat et al. 2020; Tiwari et al. 2017), decision makings (Cagman and Enginoglu 2010), and graph theory (Ali 
et al. 2017). Later in 2003, a fuzzy soft set was promoted by Maji et al. (2003) by combining the concept of fuzzy sets (Zadeh 1965) and soft sets (Molodtsov 1999). To explore the routine life issues, a huge number of different types of uncertainties have been judged by fuzzy soft sets (Roy and Maji 2007), and it has numerous applications to hand out with parameterizations and granularity (Cagman et al. 2010; Chen et al. 2005; Pei and Miao 2005).

The different portrait of it known as intuitionistic fuzzy sets (IFSs) was introduced by Bulgarian mathematician Atanassov (1986). By the property of the vagueness of IFSs, for considering unsure data, some researchers work to integrate it with influencing learning methods for better results (Garg and Kumar 2018; Liu et al. 2020; Xu and Yager 2006; Xu 2007). Notedly, aggregation operators (Xu and Yager 2006; Xu 2007), distance measures (Szmidt and Kacprzyk 2000; Wang and Xin 2005) and similarity measures (Dengfeng and Chuntian 2001; Fei et al. 2019; Liang and Shi 2003) are main operations to deal with vague information. The practical setting from IFSs is parameterized, and it appeared as intuitionistic fuzzy soft sets (or IFSSs) (Maji et al. 2001b). It found a huge number of applications in recent years (Akram and Shahzadi 2018; Bashir et al. 2012; Das and Kar 2014; Garg and Arora 2018a; Yin et al. 2012).

For ambiguous problems where IFSSs are not sufficient Agarwal investigated an extra input-based structure called generalized intuitionistic fuzzy soft sets (or GIFSSs) (Agarwal et al. 2013), its supplementary multi-criteria decision making (MCDM) was proposed by Garg and Arora (2018b); Selvachandran et al. (2017). Another unambiguous shape of GIFSSs which clarify the different meanings of opinions of senior persons on underlying IFSS is pictured by Feng et al. (2019) and also see Hayat et al. (2018). More correctly the group-based GIFSS was proposed by Hayat et al. (2018b) and recently various applications of it have been deduced Khan et al. (2020a, b).

\subsection{Motivations}

The concept of GIFSS is a perspective model to define another opinion over IFSS regarding assessments and judgments of moderators (Agarwal et al. 2013). But in Agarwal's model of GIFSS, the extra input over IFSS was turned into another IFSS. The additional information seemed like a group of values rather than IFSs. After that, Khalil pointed out errors in the original concept of GIFSSs, and he gave a note on it (Khalil 2015). Thereafter, captious insight into GIFSSs was needed to determine the adequacy of the definition. In 2019, Feng gave a reformation of the definition of GIFSS (Feng et al. 2019). From this new perspective, a number of new operations for enhancing both theoretical and practical aspects of GIFSSs, such as complement, extended/restricted unions and extended/restricted intersections have been investigated (Feng et al. 2019; Hayat et al. 2018b).

The precise practical development of GIFSSs is faculty appointment problem using a scorified method (Feng et al. 2019) and finest design evaluation in design systems for a new product using AND-operation (Hayat et al. 2018). Feng's GIFSS was not enough to determine more than one assessment of moderators. On this motivation, Hayat enumerated IFSs of moderators with IFSS (or data obtained by a committee of experts) which gives GGIFSSs (Hayat et al. 2018b). To the best of our knowledge, Hayat's GGIFSS is a primary form of GIFSS and it is an unquestionably dominance notion (Hayat et al. 2018b).

In fact, with appropriate notions, an appropriate method containing a favorable mechanism is a root point in MCDM. In order to mechanize GGIFSS with MCDM, Hayat et al. (2018b) made use of the ancient ideas of operators (Xia et al. 2013; Xu and Yager 2006; Xu 2007). Normally, the most precise assembling process compels the nature of MCDM systems which assembles reliance data and acts in a compressed manner. In recent few years, aggregation operators on IFSS or GIFSS have been investigated but a lot of improvement is needed to obtain more and more accurate results. Hayat's GGIFSS-based aggregation operators (Hayat et al. 2018b) are one aspect of compelling IF information but a more approved form was required which can manage all components of GGIFSS in a very linear way. Thus an aggregation system was necessary to get precise aggregation on both IFSS and IF in GGIFSS.

Our study develops some new generalized weighted averaging (GWA) aggregation operators and generalized weighted geometric (GWG) aggregation operators acting on GGIFSS. We will provide several properties of the new operators. By advantages of new results, we will perform multi-attribute decision making on GIFSSs and their applications in real life. Finally, we will show the superiorities of our method over major existing methods.

\section{Preliminaries}

This section recalls some terminologies and results related to fuzzy sets, soft sets and IFSs.

\subsection{Fuzzy sets}

Zadeh founded fuzzy set theory based on the concept of partial membership, which allows researchers to constitute a new framework for incorporating uncertainty and vagueness.

Definition 2.1 (Zadeh 1965) The fuzzy set (or FS) $\mathcal{P}$ is usually identified as $\mathcal{P}=\left\{\left(a, \mu_{\mathcal{P}}(a)\right) \mid a \in X\right\}$ where $\mu_{\mathcal{P}}: X \rightarrow$ $[0,1]$ is the degree of membership. 


\subsection{Intuitionistic fuzzy sets}

The FS was insufficient to represent non-credibility information in some real-life situations. Atanassov proposed the concept of an intuitionistic fuzzy set (IFS) to get over certain drawbacks of Zadeh's FSs. Notwithstanding, Atanassov's IFSs offer credible degree $\mu$ and non-credible degree $v$ for each element of a set. This was not the case for traditional fuzzy sets. Although the property $\mu+v \leq 1$ is imposed, it can hold with strict inequality $\mu+v<1$ because the degree of non-credibility given by $v$ is not necessarily equal to $\mu^{c}$. Thus any FS can be interpreted as an IFS whenever $v=1-\mu$. The formal expression of IFSs is given in the following way:

Definition 2.2 (Atanassov 1986) An IFS $\mathcal{P}$ comprising a set $X$ is expressed as:

$\mathcal{P}=\left\{\left(a, \mu_{\mathcal{P}}(a), \nu_{\mathcal{P}}(a)\right), \forall a \in X\right\}$

where $0 \leq \mu_{\mathcal{P}}(a) \leq 1,0 \leq \nu_{\mathcal{P}}(a) \leq 1$ and $\mu_{\mathcal{P}}(a)+$ $v_{\mathcal{P}}(a) \leq 1$. We denote this pair as $\mathcal{P}=\left\langle a, \mu_{\mathcal{P}}, v_{\mathcal{P}}\right\rangle$ and called as an IF value(IFV). The set of all IFSs over $X$ is denoted by IFS(X).

The main factor included in an IFS is its hesitancy, and it is contemplated as follow:

Definition 2.3 (Atanassov 1986, 1999) A grade over credible and non-credible degrees is defined by

$\pi_{\mathcal{P}}(a)=1-\left(\mu_{\mathcal{P}}(a)+v_{\mathcal{P}}(a)\right) \forall a \in X$

and it expressed the degree of hesitancy of $a$ to $\mathcal{P}$. Where $\mu_{\mathcal{P}}(a)$ is called credible degree and $\nu_{\mathcal{P}}(a)$ is called noncredible degree.

For any contrary $a \in X,\left\langle\mu_{\mathcal{P}}(a), \nu_{\mathcal{P}}(a)\right\rangle$ is a simplified form of IFV. For more on IFVs see Feng et al. (2019), Hayat et al. (2018) and Deschrijver and Kerre (2003) and the set of all IFVs over $X$ is expressed as $V$.

Definition 2.4 (Atanassov 1986) Suppose $\left\langle\mu_{\mathcal{P}}, v_{\mathcal{P}}\right\rangle \in V$ be an IFV. Then expectation account value or score of $\left\langle\mu_{\mathcal{P}}, \nu_{\mathcal{P}}\right\rangle$ is a mapping from $\eta: V \rightarrow[0,1]$, indicated as follows:

$\eta_{\mathcal{P}}=\frac{\mu_{\mathcal{P}}-\nu_{\mathcal{P}}+1}{2}$

Where $\eta_{\mathcal{P}}$ is called the decision value or score value of the pair $\left\langle\mu_{\mathcal{P}}, v_{\mathcal{P}}\right\rangle$ in $\mathcal{P}$.

In contrast to the integrated information on IFVs, fundamental operations are indicated as follows:

Definition 2.5 (Xu and Yager 2006; Xu 2007) Suppose $V_{1}=$ $\left\langle\mu_{\mathcal{P}}, v_{\mathcal{P}}\right\rangle, V_{2}=\left\langle\mu_{\mathcal{P}}^{*}, v_{\mathcal{P}}^{*}\right\rangle$ be two IFVs in a universe. Then we have,
(1) $V_{1} \oplus V_{2}=\left\langle\mu_{\mathcal{P}}+\mu_{\mathcal{P}}^{*}-\mu_{\mathcal{P}} \mu_{\mathcal{P}}^{*}, v_{\mathcal{P}} v_{\mathcal{P}}^{*}\right\rangle$

(2) $V_{1} \otimes V_{2}=\left\langle\mu_{\mathcal{P}} \mu_{\mathcal{P}}^{*}, v_{\mathcal{P}}+v_{\mathcal{P}}^{*}-v_{\mathcal{P}} v_{\mathcal{P}}^{*}\right\rangle$

(3) $\epsilon V_{1}=\left\langle 1-\left(1-\mu_{\mathcal{P}}\right)^{\epsilon},\left(\nu_{\mathcal{P}}\right)^{\epsilon}\right\rangle$ where $\epsilon$ is a positive real number.

$\mathrm{Xu}$ and Yager take an insight on aggregation instruments which are given below:

Definition 2.6 (Xu and Yager 2006; Xu 2007) Suppose $c_{1}, c_{2}, c_{3}, \ldots, c_{m}$ be the intuitionistic fuzzy values (IFVs) and $\phi=\left[\phi_{1}, \phi_{2}, \phi_{3}, \ldots, \phi_{m}\right]$ be correlated weighted normalized vector, then,

$\left.\operatorname{IFWA}\left(c_{1}, c_{2}, c_{3}, \ldots, c_{m}\right) \quad\right)=\phi_{1}{ }^{c_{1}} \bigoplus \phi_{2}{ }^{c_{2}} \bigoplus \phi_{3}{ }^{c_{3}} \bigoplus, \ldots$, $\bigoplus \phi_{m}{ }^{c_{m}}=\left\langle 1-\prod_{i}^{m}\left(1-\mu_{c_{i}}\right)^{\phi_{i}}, \prod_{i}^{m} \nu_{c_{i}}^{\phi_{i}}\right\rangle$

$\operatorname{IFWG}\left(c_{1}, c_{2}, c_{3}, \ldots \quad, c_{m}\right)=c_{1}^{\phi_{1}} \otimes c_{2}^{\phi_{2}} \otimes c_{3}^{\phi_{3}} \otimes, \ldots$, $\otimes c_{m}^{\phi_{m}}=\left\langle\prod_{i}^{m} \mu_{c_{i}}^{\phi_{i}}, 1-\prod_{i}^{m}\left(1-v_{c_{i}}^{\phi_{i}}\right)\right\rangle$

where IFWA and IFWG are the IF weighted averaging and IF weighted geometric operators, respectively.

\subsection{Soft sets}

Different from fuzzy sets, a theory was built upon the basis of parameterizations by Molodtsov. It is known as the soft set theory which is built on set-valued approximate functions to show judgment into uncertain concepts by integrally considering many different scenarios expressed by parameters.

Definition 2.7 (Molodtsov 1999) Suppose $E$ be the set of parameters, $\mathcal{B} \subseteq E$. An order pair $(\mathcal{H}, \mathcal{B})$ is known as soft set on $X$, while $\mathcal{H}$ is a map expressed by $\mathcal{H}: \mathcal{B} \rightarrow P(X)$, where $P(X)$ denote the set of all subsets of $X$.

Jointly IFSs and soft sets provide IFSSs and its notion is as follows:

Definition 2.8 (Maji et al. 2001a) Suppose soft universe $(X, E)$, and $\mathcal{B} \subseteq E$. An order pair $F=(\mathcal{S}, \mathcal{B})$ is known as IFSS on $X$, while $\mathcal{S}$ is a mapping defined by $\mathcal{S}: \mathcal{B} \rightarrow$ IF $S(X)$, where $\mathcal{S}$ is indicated as an approximate-function of the IFSS $(\mathcal{S}, \mathcal{B})$.

An adjustment of influential opinion with IFSS gives a more significant model to handle uncertainty.

Definition 2.9 (Feng et al. 2019) Suppose an order pair $(X, E)$ as a soft universe, and $\mathcal{B} \subseteq E$. A triple $(\widehat{\mathcal{S}}, \mathcal{B}, \widehat{\alpha})$ is known as generalized intuitionistic fuzzy soft set (GIFSS) on $X$. The pair $(\widehat{\mathcal{S}}, \mathcal{B})$ is indicated as an fundamental IFSS on $X$ and $\widehat{\alpha}$ is indicated as a parametric IFS in $\mathcal{B}$.

This depiction of GIFSS could be more important to deal with the problems in which unsure and vague information are frequent, and it increases the precision and accuracy of outputs with the opinion of an expert in the form of IFS over a set of parameters. The more general setup of GIFSS is 
GGIFSS, and its main finding relies on more than one crucial opinions on attributes.

Definition 2.10 (Hayat et al. 2018b) Suppose an order pair $(X, E)$, as a soft universe and $\mathcal{B} \subseteq E$. A triple $(\widehat{\mathcal{S}}, \mathcal{B}, \widehat{g})$ is known as group-based generalized intuitionistic fuzzy soft set (GGIFSS) on $X$. The pair $(\widehat{\mathcal{S}}, \mathcal{B})$ is a basic IFSS (EIFSS) on $X$ and $\widehat{g}=\left\{\alpha_{1}, \alpha_{2}, \alpha_{3}, \ldots, \alpha_{p}\right\}$ where $\alpha_{1}, \alpha_{2}, \alpha_{3}, \ldots, \alpha_{p}$ are the parameterized IFSs(X) (PIFSs) of $\mathcal{B}$. We can say that " $\widehat{g}$ " is a group of PIFSs examine by " $p$ " number of experts.

The final assessments $\alpha_{d_{i^{\prime}}}\left(i^{\prime}=1,2, \ldots, p\right)$ of experts are crucial addition in IFSS such as sometime they can be sighted as customer's demands (see the case study (II) by Hayat et al. (2018b)).

\section{New aggregation operators on generalized intuitionistic fuzzy soft sets}

Hayat's GGIFSS-based aggregation operators (Hayat et al. $2018 \mathrm{~b}$ ) is one aspect of compelling IF information, but a more approved form was required which can manage all components of GGIFSS in a very linear way. Certainly, an aggregation system was necessary to get specific aggregation on both IFSS and IFSs in GGIFSS. The existing operators on GGIFSS do not entirely deal with IFVs in a GGIFSS by concerning attributes. As in decision-making, all judgments oscillate between attributes until we obtain the best alternative from the final step of a MCDM method. On this fact, here we define a new class of operators which has a great influence on many MCDM models by mean of attributes.

\subsection{Generalized weighted averaging (GWA) operators}

In contrast of GGIFSS interpreted in Definition 2.10, a tabular formulation of it must needed to investigate underlying and above cited heading.

Definition 3.1 Let $\tau_{g}=(\widehat{S}, \mathcal{B}, \widehat{g})$ be a GGIFSS over $X$ as shown in Table 1.

Where $X=\left\{\xi_{1}, \xi_{2}, \ldots, \xi_{s}\right\}$ is the set of alternatives, $\mathcal{B}=$ $\left\{v_{1}, v_{2}, \ldots, v_{n}\right\}$ is the set of attributes, and $d_{1}, d_{2}, \ldots, d_{p}$ are the set of moderators who provide their crucial assessments on IFSS. The brown part of Table 1 shows IFSS and purple part of Table 1 shows IFSs of each moderator's assessments.

Assume that $d_{0 j}^{k}=a_{k j}(j=1,2, \ldots, n, k=1,2, \ldots, s)$ and in other words $d_{0 j}^{k}$ are IFVs in brown part of Table 1. Further, $d_{i^{\prime} j}^{k}=d_{i^{\prime} j}$ for $i^{\prime}=1,2,3, \ldots, p$ and in other words $d_{i^{\prime} j}^{k}$ are IFVs in purple part of Table 1 . Let $i=0,1,2, \ldots, p$.
Table 1 GGIFSS $\tau_{g}=(\widehat{S}, \mathcal{B}, \widehat{g})$
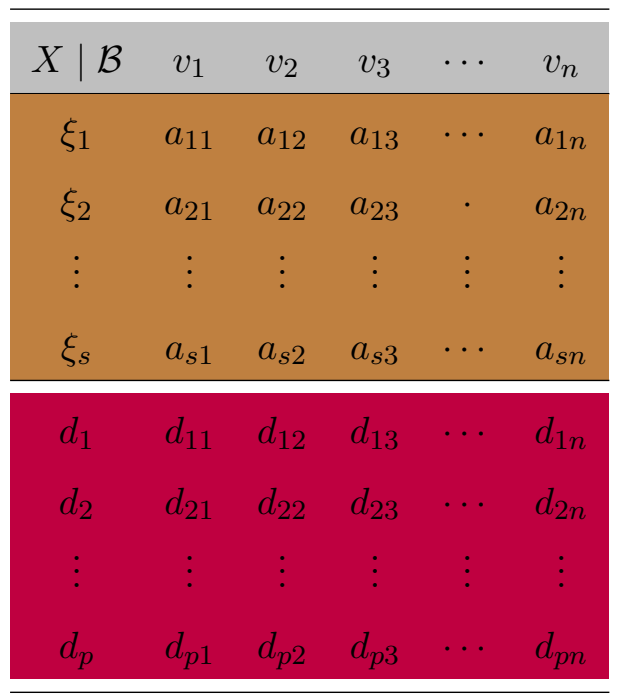

Then a characterization of IFVs in Table 1 is given by:

$d_{i j}^{k}= \begin{cases}a_{k j} & \text { if } i=0 \\ d_{i^{\prime} j} & \text { if } i>0\end{cases}$

Based on representation of GGIFSS in Table 1, the new aggregation operators are defined in following definition.

Definition 3.2 Let $\tau_{g}=(\widehat{S}, \mathcal{B}, \widehat{g})$ be a GGIFSS over $X$, given in Table 1. Let $\left[\varsigma_{1}, \varsigma_{2}, \ldots, \varsigma_{n}\right]^{T}$ be the weighted vector over set of attributes $\left\{v_{1}, v_{2}, \ldots, v_{n}\right\}$, such that $\varsigma_{j}>0$ and $\Sigma_{j=1}^{n} \varsigma_{j}=1$. Also consider weighted vector $\left[w_{0}, w_{1}, w_{2}, \ldots, w_{p}\right]^{T}$ such that $w_{i}>0$ and $\Sigma_{i=0}^{p} w_{i}=$ 1 , where $w_{1}, w_{2}, w_{3}, \ldots, w_{p}$ are the weights for assessments of senior persons/moderators and $w_{0}$ is a weight of whole data in IFSS given in Table 1 (see brown part). Note that $w_{0}$ is a weight for each IFV in IFSS. If we consider $i=0,1,2,3, \ldots, p$ then the definition of weighted generalized averaging operators (GWA) accomplished by GGIFSS is given as follows:

$$
\begin{aligned}
\delta_{k} & =W A^{G G I F S S}\left(a_{k 1}, a_{k 2}, \ldots, a_{k n}\right) \\
& =I W A_{j}\left(\begin{array}{c}
I W A_{i}\left(d_{01}^{k}, d_{11}^{k}, d_{21}^{k}, \ldots, d_{p 1}^{k}\right), \\
I W A_{i}\left(d_{02}^{k}, d_{12}^{k}, d_{22}^{k}, \ldots, d_{p 2}^{k}\right), \\
\ldots, \\
I W A_{i}\left(d_{0 n}^{k}, d_{1 n}^{k}, d_{2 n}^{k}, \ldots, d_{p n}^{k}\right)
\end{array}\right) \\
& =I W A_{j}\left(\begin{array}{c}
I W A_{i}\left(a_{k 1}, d_{11}, d_{21}, \ldots, d_{p 1}\right), \\
I W A_{i}\left(a_{k 2}, d_{12}, d_{22}, \ldots, d_{p 2}\right), \\
\ldots, \\
I W A_{i}\left(a_{k n}, d_{1 n}, d_{2 n}, \ldots, d_{p n}\right)
\end{array}\right)
\end{aligned}
$$

Where $W A^{G G I F S S}$ is called weighted averaging operators over GGIFSS, and jointly the set of all GWAs is represented as $\Delta=\left\{\delta_{1}, \delta_{2}, \ldots, \delta_{s}\right\}$. Moreover, $I W A_{i}$ is a IWA operator 
Table 2 Tabular representation of GGIFSS
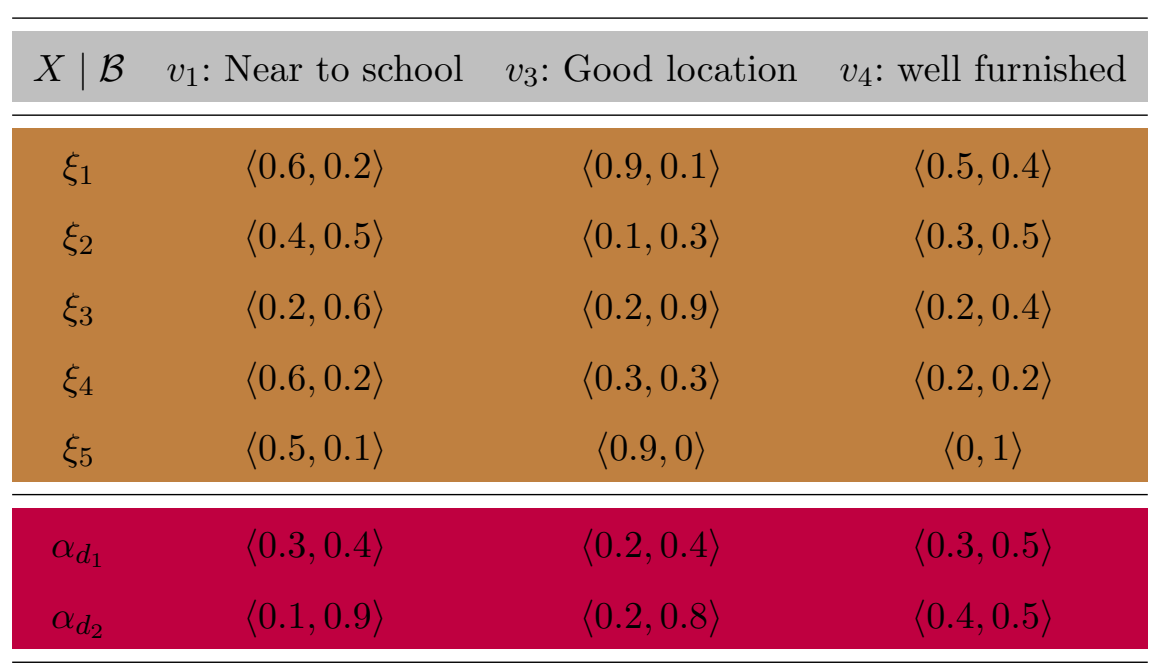

on IFS plus set of moderators and $I W A_{j}$ is IWA operator on set of parameters.

The new GWA operators aggregate the information towards attributes until the decision-IF-values of alternatives are obtained. Next, to clarify above definition, we consider an example as in the following:

Example 3.3 Let $X=\left\{\xi_{1}, \xi_{2}, \ldots, \xi_{5}\right\}$ be the set containing five houses and $E=\left\{v_{1}, v_{2}, v_{3}, v_{4}\right\}$ where $v_{i}=(i=$ $1,2,3,4)$, respectively, stands for ( "near to school," "near to hospital," "good location" and "well furnished"). Consider a set of attributes $\mathcal{B}=\left\{v_{1}, v_{3}, v_{4}\right\} \subset E$ which is desired by Mr. X and Mrs. X to buy a house. Overall assessment values (by a committee of experts) for houses concerning each attribute in $\mathcal{B}$ are given in IFSS (provided in brown part of Table 2). The demands of Mr. X and Mrs. X are given as IFSs $\alpha_{d_{1}}$ and $\alpha_{d_{2}}$, respectively (provided in purple part of Table 2). Then the GGIFSS is represented in Table 2.

Now consider the set IFVs $=\left\{a_{11}, a_{12}, a_{13}\right\}=\{\langle 0.6,0.2\rangle$, $\langle 0.9,0.1\rangle,\langle 0.5,0.4\rangle\}$ consist IFVs of house $\xi_{1}$ on attributes, respectively.

Let $w_{0}=0.29$ be the weight of whole data IFSS (provided by family of experts) and $w_{0}$ is associated with each IFV in IFSS. Also $w_{1}=0.35$ and $w_{2}=0.36$ are the weights of Mr. X and Mrs. X, respectively. Thus, we obtain a weighted vector $\left\{w_{0} / 0.29, w_{1} / 0.35, w_{2} / 0.36\right\}^{T}$. And let $\varsigma=\left\{\varsigma_{1} / 0.25, \varsigma_{2} / 0.40, \varsigma_{3} / 0.35\right\}^{T}$ be a weighted vector over given attributes. Then GWA is given by $\delta_{1}$

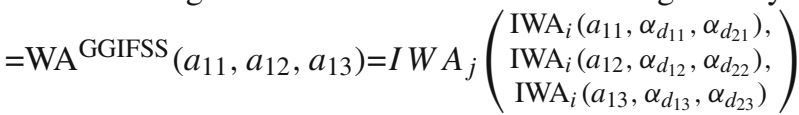

First we calculate,

$$
\begin{aligned}
& \operatorname{IWA}_{i}\left(a_{11}, \alpha_{d_{11}}, \alpha_{d_{21}}\right) \\
& \quad=\operatorname{IWA}\{\langle 0.6,0.2\rangle,\langle 0.3,0.4\rangle,\langle 0.1,0.9\rangle\}
\end{aligned}
$$

$$
\begin{aligned}
= & \left\langle 1-(1-0.6)^{0.29}(1-0.3)^{0.35}(1-0.1)^{0.36}\right. \\
= & \left.(0.2)^{0.29}(0.4)^{0.35}(0.9)^{0.36}\right\rangle
\end{aligned}
$$

Then

$$
\begin{aligned}
& \delta_{1}=\mathrm{WG}^{\mathrm{GGIFSS}}(\langle 0.6,0.2\rangle,\langle 0.9,0.1\rangle,\langle 0.5,0.4\rangle) \\
& =\operatorname{IWA}_{j}\left(\begin{array}{c}
\langle 0.348508,0.438073\rangle, \\
\langle 0.56228,0.343426\rangle \\
\langle 0.39935,0.46866\rangle
\end{array}\right) \\
& =\left\langle\begin{array}{c}
1-(1-0.348508)^{0.25}(1-0.56228)^{0.40}(1-0.39935)^{0.35}, \\
(0.438073)^{0.25}(0.343426)^{0.40}(0.46866)^{0.35}
\end{array}\right\rangle \\
& =\langle 0.4599010,0.4069328\rangle
\end{aligned}
$$

Similarly, we can calculate $\delta_{2}, \delta_{3}, \delta_{4}$ and $\delta_{5}$.

Theorem 1 Let $a_{k j}=\left\langle\mu_{k j}, v_{k j}\right\rangle$ and $d_{i^{\prime} j}=\left\langle\mu_{i^{\prime} j}, v_{i^{\prime} j}\right\rangle$ be the IFVs, where $k=1,2,3, \ldots, s, j=1,2,3 \ldots, n$ and $i^{\prime}=1,2,3 \ldots, p$. If we consider $i=0,1,2,3, \ldots, p$ then GWA operator is given by $W A^{G G I F S S}\left(a_{k 1}, a_{k 2}, \ldots, a_{k n}\right)=$ $\left\langle 1-\prod_{j=1}^{n}\left(1-\left(1-\prod_{i=0}^{p}\left(1-\mu_{d_{i j}^{k}}\right)^{w_{i}}\right)\right)^{\varsigma_{j}}, \prod_{j=1}^{n}\left(\prod_{i=0}^{p}\right.\right.$ $\left.\left.\left(v_{d_{i j}^{k}}\right)^{w_{i}}\right)^{\varsigma_{j}}\right\rangle$.

Proof Suppose $p=1$ and $n=2$. First of all, for $k=1$ we use mathematical induction on $n$, we have

$\mathrm{WA}^{\operatorname{GGIFSS}}\left(a_{11}, a_{12}\right)=\operatorname{IFWA}_{j}\left(\begin{array}{c}\operatorname{IWA}_{i}\left(d_{01}^{1}, d_{11}^{1}\right), \\ \operatorname{IWA}_{i}\left(d_{02}^{1}, d_{12}^{1}\right)\end{array}\right)$ 


$$
=\operatorname{IFWA}_{j}\left(\begin{array}{c}
\operatorname{IWA}_{i}\left(a_{11}, d_{11}\right) \\
\operatorname{IWA}_{i}\left(a_{12}, d_{12}\right)
\end{array}\right)
$$

Now, $\operatorname{IWA}_{i}\left(a_{11}, d_{11}\right)=\left\langle 1-\left(1-\mu_{a_{11}}\right)^{w_{0}} \cdot\left(1-\mu_{d_{11}}\right)^{w_{1}}, v_{a_{11}}^{w_{0}}\right.$. $\left.v_{d_{11}}^{w_{1}}\right\rangle \operatorname{IWA}_{i}\left(a_{12}, d_{12}\right)=\left\langle 1-\left(1-\mu_{a_{12}}\right)^{w_{0}} \cdot\left(1-\mu_{d_{12}}\right)^{w_{1}}, v_{a_{12}}^{w_{0}}\right.$. $\left.v_{d_{12}}^{w_{1}}\right\rangle$ Thus,

$$
\begin{aligned}
& \mathrm{WA}^{\mathrm{GGIFSS}}\left(a_{11}, a_{12}\right) \\
& =\operatorname{IFWA}_{j}\left(\begin{array}{c}
\left\langle 1-\left(1-\mu_{a_{11}}\right)^{w_{0}} \cdot\left(1-\mu_{d_{11}}\right)^{w_{1}},\right. \\
\left.v_{a_{11}}^{w_{0}} \cdot v_{d_{11}}^{w_{1}}\right\rangle, \\
\left\langle 1-\left(1-\mu_{a_{12}}\right)^{w_{0}} \cdot\left(1-\mu_{d_{12}}\right)^{w_{1}},\right. \\
\left.v_{a_{12}}^{w_{0}} \cdot v_{d_{12}}^{w_{1}}\right\rangle
\end{array}\right) \\
& =\left(\begin{array}{c}
1-\left(\left(1-\left(1-\left(1-\mu_{a_{11}}\right)^{w_{0}} \cdot\left(1-\mu_{d_{11}}\right)^{w_{1}}\right)\right)^{\varsigma_{1}} \cdot\right. \\
\left.\left(1-\left(1-\left(1-\mu_{a_{12}}\right)^{w_{0}} \cdot\left(1-\mu_{d_{12}}\right)^{w_{1}}\right)\right)^{\varsigma_{2}}\right), \\
\left(v_{a_{11}}^{w_{0}} \cdot v_{d_{11}}^{w_{1}}\right)^{\varsigma_{1}} \cdot\left(v_{a_{12}}^{w_{0}} \cdot v_{d_{12}}^{w_{1}}\right)^{\varsigma_{2}}
\end{array}\right) \\
& =\left(\begin{array}{c}
1-\left(\left(1-\left(1-\left(1-\mu_{d_{01}^{1}}\right)^{w_{0}} \cdot\left(1-\mu_{d_{11}^{1}}\right)^{w_{1}}\right)\right)^{\varsigma 1} \cdot\right. \\
\left.\left(1-\left(1-\left(1-\mu_{d_{02}^{1}}\right)^{w_{0}} \cdot\left(1-\mu_{d_{12}^{1}}\right)^{w_{1}}\right)\right)^{\varsigma_{2}}\right), \\
\left(v_{d_{01}^{1}}^{w_{0}} \cdot v_{d_{11}^{1}}^{w_{1}}\right)^{{ }^{1}} \cdot\left(v_{d_{02}^{1}}^{w_{0}} \cdot v_{d_{12}^{1}}^{w_{1}}\right)^{\varsigma_{2}}
\end{array}\right) \\
& =\left(\begin{array}{c}
1-\left(1-\left(1-\left(\prod_{i=0}^{1}\left(1-\mu_{d_{i 1}^{1}}\right)^{w_{i}}\right)\right)^{\varsigma 1}\right) . \\
\left(1-\left(1-\left(\prod_{i=0}^{1}\left(1-\mu_{d_{i 2}^{1}}\right)^{w_{i}}\right)\right)^{\varsigma_{2}}\right), \\
\left(\left(\prod_{i=0}^{1}\left(v_{d_{i 1}^{1}}\right)^{w_{i}}\right)^{\varsigma_{1}} \cdot\left(\prod_{i=0}^{1}\left(v_{d_{i 2}^{1}}\right)^{w_{i}}\right)^{\varsigma_{2}}\right)
\end{array}\right) \\
& =\left(\begin{array}{c}
1-\prod_{j=1}^{2}\left(1-\left(1-\left(\prod_{i=0}^{1}\left(1-\mu_{d_{i j}^{1}}\right)^{w_{i}}\right)\right)\right)^{\varsigma_{j}}, \\
\prod_{j=1}^{2}\left(\prod_{i=0}^{1}\left(v_{d_{i j}^{1}}\right)^{w_{i}}\right)^{\varsigma_{j}}
\end{array}\right)
\end{aligned}
$$

Hence the theorem is valid for $n=2$. Considering that this result is fine for $n=n^{\prime}$ that is $\mathrm{WA}^{\mathrm{GGIFSS}}\left(a_{k 1}, a_{k 2}, \ldots, a_{k n^{\prime}}\right)$ $=\left\langle 1-\prod_{j=1}^{n^{\prime}}\left(1-\left(1-\prod_{i=0}^{p}\left(1-\mu_{d_{i j}^{k}}\right)^{w_{i}}\right)\right)^{\varsigma_{j}}, \prod_{j=1}^{n^{\prime}}\right.$ $\left.\left(\prod_{i=0}^{p}\left(v_{d_{i j}^{k}}\right)^{w_{i}}\right)^{\varsigma_{j}}\right\rangle$. Then for $n=n^{\prime}+1, W A^{G G I F S S}$ $\left(a_{k 1}, a_{k 2}, \ldots, a_{k\left(n^{\prime}+1\right)}\right)=\left\langle 1-\prod_{j=1}^{\left(n^{\prime}+1\right)}\left(1-\left(1-\prod_{i=0}^{p}(1-\right.\right.\right.$ $\left.\left.\left.\left.\mu_{d_{i j}^{k}}\right)^{w_{i}}\right)\right)^{\varsigma_{j}}, \prod_{j=1}^{\left(n^{\prime}+1\right)}\left(\prod_{i=0}^{p}\left(v_{d_{i j}^{k}}\right)^{w_{i}}\right)^{\varsigma_{j}}\right\rangle$. So by mathematical induction Theorem 1 satisfies for all positive integer $n$.

Theorem 2 Idempotency: If $a_{k j}=a_{j}=a$ and $d_{i^{\prime} j}=d_{i^{\prime}}=$ a for all $j=1,2, \ldots, n$ then $W A^{G G I F S S}\left(a_{k 1}, a_{k 2}, \ldots, a_{k n}\right)$ $=a$.

Proof Given $a_{k j}=a_{j}=a$ and $d_{i^{\prime} j}=d_{i^{\prime}}=a$ for all $j=1,2, \ldots, n, i^{\prime}=1,2, \ldots, p$. Then,

$$
\begin{aligned}
& \text { WA }^{\mathrm{GGIFSS}}\left(a_{k 1}, a_{k 2}, \ldots, a_{k n}\right) \\
& =\left\langle\begin{array}{c}
1-\prod_{j=1}^{n}\left(1-\left(1-\prod_{i=0}^{p}\left(1-\mu_{d_{i j}^{k}}\right)^{w_{i}}\right)\right)^{\varsigma_{j}}, \\
\prod_{j=1}^{n}\left(\prod_{i=0}^{p}\left(v_{d_{i j}^{k}}\right)^{w_{i}}\right)^{\varsigma_{j}}
\end{array}\right\rangle \\
& =\left\langle\begin{array}{c}
1-\prod_{j=1}^{n}\left(1-\left(1-\left(1-\mu_{a}\right)^{\sum_{i=0}^{p} w_{i}}\right)\right)^{\varsigma_{j}}, \\
\prod_{j=1}^{n}\left(\left(v_{a}\right)^{\sum_{i=0}^{p} w_{i}}\right)^{\varsigma_{j}}
\end{array}\right\rangle \\
& =\left\langle 1-\prod_{j=1}^{n}\left(1-\left(1-\left(1-\mu_{a}\right)\right)\right)^{\varsigma_{j}}, \prod_{j=1}^{n}\left(\left(v_{a}\right)\right)^{\varsigma_{j}}\right\rangle
\end{aligned}
$$

$$
\begin{aligned}
& =\left\langle 1-\prod_{j=1}^{n}\left(1-\mu_{a}\right)^{\varsigma_{j}}, \prod_{j=1}^{n}\left(v_{a}\right)^{\varsigma_{j}}\right\rangle \\
& =\left\langle 1-\left(1-\mu_{a}\right)^{\sum_{j=1}^{n} \varsigma_{j}},\left(v_{a}\right)^{\sum_{j=1}^{n} \varsigma_{j}}\right\rangle \\
& =\left\langle 1-\left(1-\mu_{a}\right), v_{a}\right\rangle \\
& =\left\langle\mu_{a}, v_{a}\right\rangle \\
& =a
\end{aligned}
$$

Hence WA ${ }^{\mathrm{GGIFSS}}\left(a_{k 1}, a_{k 2}, \ldots, a_{k n}\right)=a$.

Theorem 3 Boundedness: If $d_{j}^{+}=\left\langle\left(\mu_{\left(d_{i j}^{k}\right)}\right)^{\max },\left(v_{\left(d_{i j}^{k}\right)}\right)^{\min }\right\rangle$ and $d_{j}^{-}=\left\langle\left(\mu_{\left(d_{i j}^{k}\right)}\right)^{\min },\left(v_{\left(d_{i j}^{k}\right)}\right)^{\max }\right\rangle \forall k=1,2, \ldots, s$ and $i=0,1,2, \ldots$, pthend $d_{j}^{-} \leq W A^{G G I F S S}\left(a_{k 1}, a_{k 2}, \ldots, a_{k n}\right)$ $\leq d_{j}^{+}$.

Proof Let $d_{i j}^{k}=\left(\mu_{i j}^{k}, v_{i j}^{k}\right) \forall i, j, k$ be IFVs in GGIFSS. Since $\left(\mu_{\left(d_{i j}^{k}\right)}\right)^{\min } \leq \mu_{\left(d_{i j}^{k}\right)} \leq\left(\mu_{\left(d_{i j}^{k}\right)}\right)^{\max } \Longrightarrow 1-$ $\left(\mu_{\left(d_{i j}^{k}\right)}\right)^{\min } \geq 1-\mu_{\left(d_{i j}^{k}\right)} \geq 1-\left(\mu_{\left(d_{i j}^{k}\right)}\right)^{\max } \Longrightarrow$ $\prod_{i=0}^{p}\left(1-\left(\mu_{\left(d_{i j}^{k}\right)}\right)^{\mathrm{min}}\right)^{w_{i}} \geq \prod_{i=0}^{p}\left(1-\mu_{\left(d_{i j}^{k}\right)}\right)^{w_{i}} \geq \prod_{i=0}^{p}(1-$ $\left.\left(\mu_{\left(d_{i j}^{k}\right)}\right)^{\max }\right)^{w_{i}} \Longrightarrow\left(1-\left(\mu_{\left(d_{i j}^{k}\right)}\right)^{\min }\right)^{\sum_{i=0}^{p} w_{i}=1} \geq \prod_{i=0}^{p}(1-$ $\left.\mu_{\left(d_{i j}^{k}\right)}\right)^{w_{i}} \geq\left(1-\left(\mu_{\left(d_{i j}^{k}\right)}\right)^{\max }\right)^{\sum_{i=0}^{p} w_{i}=1} \quad \Longrightarrow \quad(1-$ $\left.\left(\mu_{\left(d_{i j}^{k}\right)}\right)^{\min }\right) \geq \prod_{i=0}^{p}\left(1-\mu_{\left(d_{i j}^{k}\right)}\right)^{w_{i}} \geq\left(1-\left(\mu_{\left(d_{i j}^{k}\right)}\right)^{\max }\right) \Longrightarrow$ $1-\left(1-\left(\mu_{\left(d_{i j}^{k}\right)}\right)^{\mathrm{min}}\right) \leq 1-\prod_{i=0}^{p}\left(1-\mu_{\left(d_{i j}^{k}\right)}\right)^{w_{i}} \leq 1-(1-$ $\left.\left(\mu_{\left(d_{i j}^{k}\right)}\right)^{\max }\right) \Longrightarrow\left(\mu_{\left(d_{i j}^{k}\right)}\right)^{\min } \leq 1-\prod_{i=0}^{p}\left(1-\mu_{\left(d_{i j}^{k}\right)}\right)^{w_{i}} \leq$ $\left(\mu_{\left(d_{i j}^{k}\right)}\right)^{\max } \Longrightarrow \prod_{j=0}^{n}\left(1-\left(\mu_{\left(d_{i j}^{k}\right)}\right)^{\min }\right)^{\varsigma_{j}} \geq \prod_{j=0}^{n}(1-(1-$ $\left.\left.\prod_{i=0}^{p}\left(1-\mu_{\left(d_{i j}^{k}\right)}\right)^{w_{i}}\right)\right)^{\varsigma_{j}} \geq \prod_{j=0}^{n}\left(1-\left(\mu_{\left(d_{i j}^{k}\right)}\right)^{\max }\right)^{\varsigma_{j}} \Longrightarrow$

$$
\left(1-\left(\mu_{\left(d_{i j}^{k}\right)}\right)^{\min }\right)^{\sum_{j=0}^{n} S_{j}=1} \geq \prod_{j=0}^{n}\left(1-\left(1-\prod_{i=0}^{p}(1-\right.\right.
$$
$\left.\left.\left.\mu_{\left(d_{i j}^{k}\right)}\right)^{w_{i}}\right)\right)^{\varsigma_{j}} \geq\left(1-\left(\mu_{\left(d_{i j}^{k}\right)}\right)^{\max }\right)^{\sum_{j=0}^{n} \varsigma_{j}=1} \Longrightarrow$

$\left(1-\left(\mu_{\left(d_{i j}^{k}\right)}\right)^{\mathrm{min}}\right) \geq \prod_{j=0}^{n}\left(1-\left(1-\prod_{i=0}^{p}\left(1-\mu_{\left(d_{i j}^{k}\right)}\right)^{w_{i}}\right)\right)^{\varsigma_{j}} \geq$ $\left(1-\left(\mu_{\left(d_{i j}^{k}\right)}\right)^{\max }\right) \Longrightarrow 1-\left(1-\left(\mu_{\left(d_{i j}^{k}\right)}\right)^{\min }\right) \leq 1-\prod_{j=0}^{n}(1-$ $\left.\left(1-\prod_{i=0}^{p}\left(1-\mu_{\left(d_{i j}^{k}\right)}\right)^{w_{i}}\right)\right)^{\varsigma_{j}} \leq 1-\left(1-\left(\mu_{\left(d_{i j}^{k}\right)}\right)^{\max }\right) \Longrightarrow$ $\left(\mu_{\left(d_{i j}^{k}\right)}\right)^{\min } \leq 1-\prod_{j=0}^{n}\left(1-\left(1-\prod_{i=0}^{p}\left(1-\mu_{\left(d_{i j}^{k}\right)}\right)^{w_{i}}\right)\right)^{\varsigma_{j}} \leq$ $\left(\mu_{\left(d_{i j}^{k}\right)}\right)^{\max }$.

In similar way we can prove $\left(v_{\left(d_{i j}^{k}\right)}\right)^{\min } \leq$ $\prod_{j=0}^{n}\left(\prod_{i=0}^{p}\left(v_{\left(d_{i j}^{k}\right)}\right)^{w_{i}}\right)^{\varsigma_{j}} \leq\left(v_{\left(d_{i j}^{k}\right)}\right)^{\max }$.

This concludes the proof of the theorem, that is,

$d_{j}^{-} \leq \mathrm{WA}^{\mathrm{GGIFSS}}\left(a_{k 1}, a_{k 2}, \ldots, a_{k n}\right) \leq d_{j}^{+}$.

Theorem 4 Monotonicity: If $d_{i j}^{\prime k}$ and $d_{i j}^{k}$ are two IFVs such that $d_{i j}^{\prime k} \leq d_{i j}^{k}$ then, WA GGIFSS $\left(a_{k 1}^{\prime}, a_{k 2}^{\prime}, \ldots, a_{k n}^{\prime}\right) \leq$ $W A^{G G I F S S}\left(a_{k 1}, a_{k 2}, \ldots, a_{k n}\right)$. 
Proof It can be concluded from Theorem 3.

Proposition 3.4 Let $\tau_{g}=(\widehat{S}, A, \widehat{g})$ be a GGIFSS, given in Table 1. Then,

(1) If $d_{i^{\prime} j}=\langle 1,0\rangle$ for all $i^{\prime}$ and $j$, then $\Delta=\{\langle 1,0\rangle,\langle 1,0\rangle$, $\ldots,\langle 1,0\rangle\}$.

(2) If $d_{0 j}^{k}=a_{k j}=\langle 1,0\rangle$ for all $j$ and $k$, then $\Delta=$ $\{\langle 1,0\rangle,\langle 1,0\rangle, \ldots,\langle 1,0\rangle\}$.

(3) If $d_{i^{\prime} j}=\langle 1,0\rangle$ for all $i^{\prime}, j$ and $d_{0 j}^{k}=a_{k j}=\langle 1,0\rangle$ for all $j, k$, then $\Delta=\{\langle 1,0\rangle,\langle 1,0\rangle, \ldots,\langle 1,0\rangle\}$.

(4) If $d_{i^{\prime} j}=\langle 0,1\rangle$ for all $i^{\prime}, j$ and $d_{0 j}^{k}=a_{k j}=\langle 0,1\rangle$ for all $j$, $k$, then $\Delta=\{\langle 0,1\rangle,\langle 0,1\rangle, \ldots,\langle 0,1\rangle\}$.

Proof Straightforward.

\subsection{Generalized weighted geometric (GWG) operators}

In this section, GWG operators are computed and related axioms are investigated.

Definition 3.5 Let $\tau_{g}=(\widehat{S}, \mathcal{B}, \widehat{g})$ be a GGIFSS over $X$, given in Table 1. Let $\left[\varsigma_{1}, \varsigma_{2}, \ldots, \varsigma_{n}\right]^{T}$ be the weighted vector over set of attributes $\left\{v_{1}, v_{2}, v_{3}, \ldots, v_{n}\right\}$, such that $\varsigma_{j}>0$ and $\Sigma_{j=1}^{n} \varsigma_{j}=1$. Also consider weighted vector $\left[w_{0}, w_{1}, w_{2}, \ldots, w_{p}\right]^{T}$ such that $w_{i}>0$ and $\Sigma_{i=0}^{p} w_{i}=1$, where $w_{1}, w_{2}, w_{3}, \ldots, w_{p}$ are the weights for assessments of senior persons and $w_{0}$ is a weight of whole data in IFSS given in Table 1 (see brown part). If we consider $i=0,1,2,3, \ldots, p$ then the definition of weighted averaging operators (GWG) accomplished by GGIFSS is given as follows:

$$
\begin{aligned}
& \operatorname{WG}^{\mathrm{GGIFSS}_{(}}\left(a_{k 1}, a_{k 2}, \ldots, a_{k n}\right) \\
& =\mathrm{IWG}_{j}\left(\begin{array}{c}
\operatorname{IWG}_{i}\left(d_{o 1}^{k}, d_{11}^{k}, d_{21}^{k}, \ldots, d_{p 1}^{k}\right), \\
I W G_{i}\left(d_{o 2}^{k}, d_{12}^{k}, d_{22}^{k}, \ldots, d_{p 2}^{k}\right), \\
\ldots, \\
\operatorname{IWG}_{i}\left(d_{o n}^{k}, d_{1 n}^{k}, d_{2 n}^{k}, \ldots, d_{p n}^{k}\right)
\end{array}\right) \\
& =\operatorname{IWG}_{j}\left(\begin{array}{c}
\operatorname{IWG}_{i}\left(a_{k 1}, d_{11}, d_{21}, \ldots, d_{p 1}\right), \\
I W G_{i}\left(a_{k 2}, d_{12}, d_{22}, \ldots, d_{p 2}\right), \\
\ldots, \\
\operatorname{IWG}_{i}\left(a_{k n}, d_{1 n}, d_{2 n}, \ldots, d_{p n}\right)
\end{array}\right)
\end{aligned}
$$

where $\mathrm{WG}^{\mathrm{GGIFSS}}$ is called weighted geometric operators over GGIFSS, and jointly the set of all GWGs is represented as $\Delta^{\prime}=\left\{\delta_{1}^{\prime}, \delta_{2}^{\prime}, \ldots, \delta_{s}^{\prime}\right\}$. Moreover, $\mathrm{IWG}_{i}$ is a IWG operator on IFS plus set of moderators and $\mathrm{IWG}_{j}$ is IWG operator on set of parameters.

Next an example is considered to clarify above notion.

Example 3.6 Consider Example 3.3, where $\operatorname{IFV}\left(\xi_{4}\right)=$ $\left\{a_{41}, a_{42}, a_{43}\right\}=\{\langle 0.6,0.2\rangle,\langle 0.3,0.3\rangle,\langle 0.2,0.2\rangle\}$.
Consider same weighted vectors as given in Example 3.3. Then GWG is given by $\delta_{4}^{\prime}=\mathrm{WG}^{\mathrm{GGIFSS}}\left(a_{41}, a_{42}, a_{43}\right)=\mathrm{IWG}_{j}$ $\left(\begin{array}{c}\operatorname{IWG}_{i}\left(a_{41}, \alpha_{d_{11}}, \alpha_{d_{21}}\right), \\ \operatorname{IWG}_{i}\left(a_{42}, \alpha_{d_{12}}, \alpha_{d_{22}}\right), \\ \operatorname{IWG}_{i}\left(a_{43}, \alpha_{d_{13}}, \alpha_{d_{23}}\right)\end{array}\right)$

First we calculate,

$$
\begin{aligned}
& \operatorname{IWG}_{i}\left(a_{41}, \alpha_{d_{11}}, \alpha_{d_{21}}\right) \\
& =\operatorname{IWG}\{\langle 0.6,0.2\rangle,\langle 0.3,0.4\rangle,\langle 0.1,0.9\rangle\} \\
& =\left\langle\begin{array}{c}
(0.6)^{0.29}(0.3)^{0.35}(0.6)^{0.36} \\
1-(1-0.2)^{0.29}(1-0.4)^{0.35}(1-0.9)^{0.36}
\end{array}\right\rangle \\
& =\langle 0.4707,0.5134\rangle
\end{aligned}
$$

$\operatorname{IWG}_{i}\left(a_{42}, \alpha_{d 12}, \alpha_{d 22}\right)=\operatorname{IWG}\{\langle 0.3,0.3\rangle,\langle 0.2,0.4\rangle,\langle 0.2$, $0.8\rangle\}=\langle 0.2249,0.5775\rangle \mathrm{IWG}_{i}\left(a_{43}, \alpha_{d 13}, \alpha_{d 23}\right)=\mathrm{IWG}$ $\{\langle 0.2,0.2\rangle,\langle 0.3,0.5\rangle,\langle 0.4,0.5\rangle\}=\langle 0.2958,0.4269\rangle$.

Then

$$
\begin{aligned}
\delta_{4}^{\prime} & =\mathrm{WG}^{\mathrm{GGIFSS}_{(}}{ }_{(\langle 0.6,0.2\rangle,\langle 0.3,0.3\rangle,\langle 0.2,0.2\rangle)} \\
& =\mathrm{IWG}_{j}\left(\begin{array}{c}
\langle 0.4707,0.5134\rangle, \\
\langle 0.2249,0.5775\rangle, \\
\langle 0.2958,0.4269\rangle
\end{array}\right) \\
& =\left\langle\begin{array}{c}
(0.4707)^{0.25}(0.2249)^{0.40}(0.2958)^{0.35}, \\
1-(1-0.5134)^{0.25}(1-0.5775)^{0.40}(1-0.4269)^{0.35}
\end{array}\right\rangle \\
& =\langle 0.2977,0.5130\rangle
\end{aligned}
$$

Similarly, we can calculate $\delta_{1}, \delta_{2}, \delta_{3}$ and $\delta_{5}$.

Theorem 5 If $a_{k j}=\left\langle\mu_{k j}, v_{k j}\right\rangle$ and $d_{i^{\prime} j}=\left\langle\mu_{i^{\prime} j}, v_{i^{\prime} j}\right\rangle$ where $k=1,2,3, \ldots, s, j=1,2,3 \ldots, n$ and $i^{\prime}=1,2,3 \ldots, p$ be the IFVs. If we consider $i=0,1,2,3, \ldots, p$ then $G W G$ operator is given by

$W G^{G G I F S S}\left(a_{k 1}, a_{k 2}, \ldots, a_{k n}\right)=\left\langle\prod_{j=1}^{n}\left(\prod_{i=0}^{p}\left(\mu_{d_{i j}^{k}}\right)^{w_{i}}\right)^{S_{j}}\right.$, $\left.1-\prod_{j=1}^{n}\left(1-\left(1-\prod_{i=0}^{p}\left(1-v_{d_{i j}^{k}}\right)^{w_{i}}\right)\right)^{\varsigma_{j}}\right\rangle$.

Proof It is analogous to the proof of Theorem 1.

Theorem 6 Idempotency: If $a_{k j}=a_{j}=a$ and $d_{i^{\prime} j}=d_{i^{\prime}}=$ a for all $j=1,2, \ldots, n$ then $W G^{G G I F S S}\left(a_{k 1}, a_{k 2}, \ldots, a_{k n}\right)$ $=a$.

Proof It is analogous to the proof of Theorem 2.

Theorem 7 Boundedness: If $d_{j}^{+}=\left\langle\left(\mu_{\left(d_{i j}^{k}\right)}\right)^{\max },\left(v_{\left(d_{i j}^{k}\right)}\right)^{\min }\right\rangle$ and $d_{j}^{-}=\left\langle\left(\mu_{\left(d_{i j}^{k}\right)}\right)^{\min },\left(v_{\left(d_{i j}^{k}\right)}\right)^{\max }\right\rangle \forall k=1,2, \ldots, s$ and $i=0,1,2, \ldots$, p then $d_{j}^{-} \leq W G^{G G I F S S}\left(a_{k 1}, a_{k 2}, \ldots, a_{k n}\right)$ $\leq d_{j}^{+}$.

Proof It is analogous to the proof of Theorem 3.

Theorem 8 Monotonicity: If $d_{i j}^{\prime k}$ and $d_{i j}^{k}$ are two IFVs such that $d_{i j}^{\prime k} \leq d_{i j}^{k}$ then, $W G^{G G I F S S}\left(a_{k 1}^{\prime}, a_{k 2}^{\prime}, \ldots, a_{k n}^{\prime}\right) \leq$ $W G^{G G I F S S}\left(a_{k 1}, a_{k 2}, \ldots, a_{k n}\right)$. 
Proof It can be concluded from Theorem 4.

Proposition 3.7 Let $\tau_{g}=(\widehat{S}, A, \widehat{g})$ be a GGIFSS, given in Table 1. Then,

(1) If $d_{i^{\prime} j}=\langle 0,1\rangle$ for all $i^{\prime}, j$ then $\Delta^{\prime}=\{\langle 0,1\rangle,\langle 0,1\rangle, \ldots$, $\langle 0,1\rangle\}$.

(2) If $d_{0 j}^{k}=a_{k j}=\langle 0,1\rangle$ for all $j, k$ then $\Delta^{\prime}=$ $\{\langle 0,1\rangle,\langle 0,1\rangle, \ldots,\langle 0,1\rangle\}$.

(3) If $d_{i^{\prime} j}=\langle 0,1\rangle$ for all $i^{\prime}, j$ and $d_{0 j}^{k}=a_{k j}=\langle 0,1\rangle$ for all $j, k$ then $\Delta^{\prime}=\{\langle 0,1\rangle,\langle 0,1\rangle, \ldots,\langle 0,1\rangle\}$.

(4) If $d_{i^{\prime} j}=\langle 1,0\rangle$ for all $i^{\prime}, j$ and $d_{0 j}^{k}=a_{k j}=\langle 1,0\rangle$ for all $j, k$ then $\Delta^{\prime}=\{\langle 1,0\rangle,\langle 1,0\rangle, \ldots,\langle 1,0\rangle\}$.

Proof It is straightforward.

\section{Applications of new aggregation operators in MCDM}

We express application by MCDM methodology, comprising real-life situation, to highlight the usefulness of investigated aggregation operators. Initially, we show our viewpoint containing an algorithm by dint of GGIFSS and GWA or GWG operators.

\subsection{Methodology}

Consider GGIFSS in Definition 3.1, where $X=\left\{\xi_{1}, \xi_{2}, \ldots\right.$, $\left.\xi_{s}\right\}$ and $M=\left\{v_{1}, v_{2}, \ldots, v_{n}\right\}$ be the set of alternatives and attributes, respectively. GGIFSS indicates IFVs-based data, where IFSS shows the initial judgments of a committee/panel of experts on alternative $\xi_{k}(k=1,2, \ldots, s)$ and $\widehat{g}$ shows group of IFSs for each moderator's $\left(d_{1}, d_{2}, \ldots, d_{p}\right)$ assessments on IFSS.

Any IFV $r=\langle\mu(v), v(v)\rangle$ can be characterized in normalized form by:

$r=\left(\begin{array}{c}\langle\mu(v), v(v)\rangle, \text { if } v \text { is benefit criteria } \\ \langle v(v), \mu(v)\rangle, \text { if } v \text { is cost type criteria }\end{array}\right)$

Consequently, the normalized GGIFSS can be formulated as it is given in Table 1.

Let $\tau_{g}^{\prime}=\left(\widehat{S}^{\prime}, A, \widehat{g}\right)$ be the GGIFSS. Let $\left[\varsigma_{1}, \varsigma_{2}, \ldots, \varsigma_{n}\right]^{T}$ be the weighted vector over set of attributes $\left\{v_{1}, v_{2}, v_{3}, \ldots, v_{n}\right\}$, such that $\varsigma_{j}>0$ and $\Sigma_{j=1}^{n} \varsigma_{j}=1$. Also consider weighted vector $\left[w_{0}, w_{1}, w_{2}, w_{3}, \ldots, w_{p}\right]^{T}$ such that $w_{i}>0$ and $\Sigma_{i=0}^{p} w_{i}=1$, where $w_{1}, w_{2}, w_{3}, \ldots, w_{p}$ are the weights for assessments of senior persons (or senior experts or moderators) and $w_{0}$ is a weight of whole data in IFSS given in Table 1 (see brown part).

Next we calculate GWA or GWG operators over GGIFSS $\tau_{g}^{\prime}=\left(\widehat{S}^{\prime}, A, \widehat{g}\right)$. There will be $s$ number of values as $\delta_{1}$,

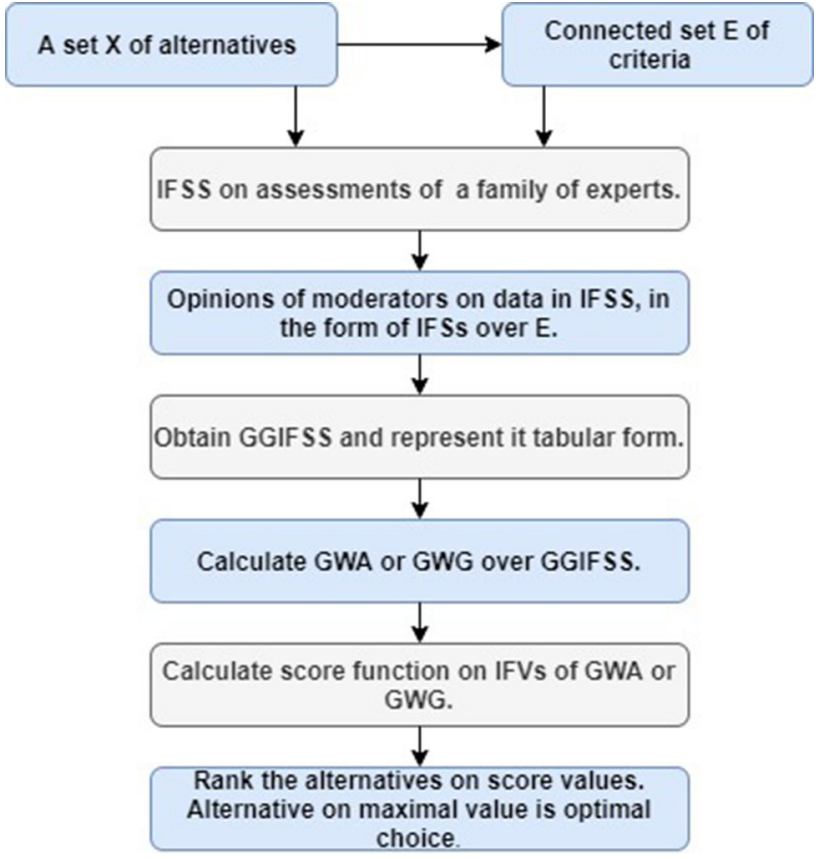

Fig. 1 A flowchart of our algorithm

$\delta_{2}, \ldots, \delta_{s}$. On each $\delta_{k}(k=1,2, \ldots, s)$ calculate the scores by using score function given in Definition 2.4. Finally we will rank the alternatives according to scores.

Consequently, we present my methodology by a systematics algorithm (see Algorithm 1) and it is depicted in Fig. 1.

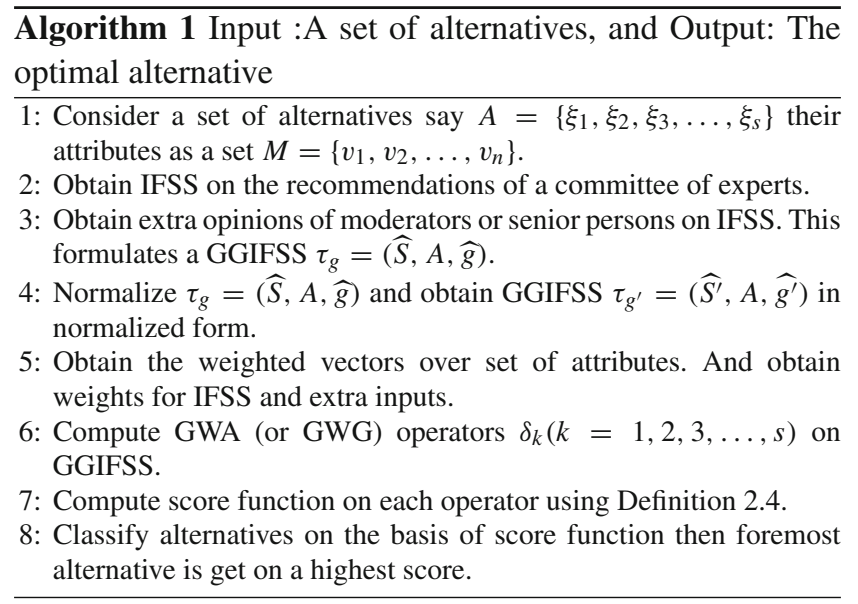

\subsection{Real-life application}

\subsubsection{Case study 1}

Hand sanitizer(HS) is considered as a simple and economical means of infection control in the recent years. It is 
Table 3 IFSS $(\widetilde{S}, E)$ in tabular form

\begin{tabular}{lllll}
\hline$X \mid E$ & $v_{1}$ & $v_{2}$ & $v_{3}$ & $v_{4}$ \\
\hline$\xi_{1}$ & $\langle 0.4,0.5\rangle$ & $\langle 0.5,0.2\rangle$ & $\langle 0.7,0.2\rangle$ & $\langle 0.4,0.3\rangle$ \\
$\xi_{2}$ & $\langle 0.6,0.2\rangle$ & $\langle 0.3,0.6\rangle$ & $\langle 0.8,0.2\rangle$ & $\langle 0.4,0.4\rangle$ \\
$\xi_{3}$ & $\langle 0.5,0.4\rangle$ & $\langle 0.4,0.4\rangle$ & $\langle 0.2,0.6\rangle$ & $\langle 0.4,0.6\rangle$ \\
$\xi_{4}$ & $\langle 0.6,0.1\rangle$ & $\langle 0.7,0.1\rangle$ & $\langle 0.5,0.1\rangle$ & $\langle 0.6,0.4\rangle$ \\
\hline
\end{tabular}

used in a wide variety of circumstances, from schools and day-care centers to hospitals and offices and from supermarkets to transportation, etc. Different firms such as the World Health Organization (WHO) and Centers for Disease Control and Prevention (CDCP) U.S. encourage the adoption of alcoholic HS. In a recent study Jing et al. (2020) recommends attributes of alcohol-based HSs as "Alcohol 60-95\%," "Hydrogen peroxide 3\%," "Glycerol and others essential oils" and "Fragrance and colorant." In markets several types of alcohol-based HSs are available but concentration and amount of above essential attributes are varied.

The fundamental aim of this application is to choose an effective alcohol-based HSs to diminish transferral of coronavirus by using the proposed operators in GGIFSS environments. Let $X=\left\{\xi_{1}, \xi_{2}, \xi_{3}, \xi_{4}\right\}$ be the set consisting of four different varieties of alcohol-based HSs. The set of attributes $E=\left\{v_{1}, v_{2}, v_{3}, v_{4}\right\}$, where

$v_{1}$ : Concentration of Alcohol

$v_{2}$ : Concentration of Hydrogen peroxide

$v_{3}$ : Quantity and quality of Glycerol and others essential oils

$v_{4}:$ Quantity of fragrance and colorant

A family of experts from a health organization gives the assessments and views for alcohol-based HSs on given attributes in the form of IFSS $(\widetilde{S}, E)$ (Table 3)

Now the two senior experts $d_{1}$ and $d_{2}$ from the health organization provide opinions on IFSS (given in Table 3 ) as IFSs,

$\alpha_{d_{1}}=\left\{v_{1} /\langle 0.4,0.6\rangle, v_{2} /\langle 0.5,0.3\rangle, v_{3} /\langle 0.5,0.4\rangle, v_{4} /\langle 0.6,0.4\rangle\right\}$,

$\alpha_{d_{2}}=\left\{v_{1} /\langle 0.5,0.4\rangle, v_{2} /\langle 0.4,0.5\rangle, v_{3} /\langle 0.5,0.3\rangle, v_{4} /\langle 0.4,0.5\rangle\right\}$,

,respectively. Then, the information can be expanded in the form of GGIFSS $\tau_{g}=(\widehat{S}, E, \widehat{g})$ given in Table 4 .

Let $w_{0}=0.37$ be the weight of whole data in IFSS (given in Table 4), $w_{1}=0.21$ and $w_{2}=0.42$ be the weights of senior experts or moderators. Thus we have general weighted vector $\left[w_{0} / 0.37, w_{1} / 0.21, w_{2} / 0.42\right]^{T}$. Let $\left[v_{1} / 0.38, v_{2} / 0.19, v_{3} / 0.21, v_{4} / 0.22\right]^{T}$ be the weighted vectors over given parameters. The GWA operator is utilized to compel data present in Table 4 , and it is
Table 4 GGIFSS $(\widehat{S}, E, \widehat{g})$ in tabular form

\begin{tabular}{lllll}
\hline$X \mid E$ & $v_{1}$ & $v_{2}$ & $v_{3}$ & $v_{4}$ \\
\hline$\xi_{1}$ & $\langle 0.4,0.5\rangle$ & $\langle 0.5,0.2\rangle$ & $\langle 0.7,0.2\rangle$ & $\langle 0.4,0.3\rangle$ \\
$\xi_{2}$ & $\langle 0.6,0.2\rangle$ & $\langle 0.3,0.6\rangle$ & $\langle 0.8,0.2\rangle$ & $\langle 0.4,0.4\rangle$ \\
$\xi_{3}$ & $\langle 0.5,0.4\rangle$ & $\langle 0.4,0.4\rangle$ & $\langle 0.2,0.6\rangle$ & $\langle 0.4,0.6\rangle$ \\
$\xi_{4}$ & $\langle 0.6,0.1\rangle$ & $\langle 0.7,0.1\rangle$ & $\langle 0.5,0.1\rangle$ & $\langle 0.6,0.4\rangle$ \\
\hline$\alpha_{d_{1}}$ & $\langle 0.4,0.6\rangle$ & $\langle 0.5,0.3\rangle$ & $\langle 0.5,0.4\rangle$ & $\langle 0.6,0.4\rangle$ \\
$\alpha_{d_{2}}$ & $\langle 0.5,0.4\rangle$ & $\langle 0.4,0.5\rangle$ & $\langle 0.5,0.3\rangle$ & $\langle 0.4,0.5\rangle$ \\
\hline
\end{tabular}

investigated as under: $\delta_{1}=\mathrm{WA}^{\mathrm{GGIFSS}}\left(a_{11}, a_{12}, a_{13}, a_{14}\right)=$ $\operatorname{IWA}_{j}\left(\begin{array}{l}\operatorname{IWA}_{i}\left(a_{11}, \alpha_{d_{11}}, \alpha_{d_{21}}\right), \\ \operatorname{IWA}_{i}\left(a_{12}, \alpha_{d_{12}}, \alpha_{d_{22}}\right), \\ \operatorname{IWA}_{i}\left(a_{13}, \alpha_{d_{13}}, \alpha_{d_{23}}\right), \\ \operatorname{IWA}_{i}\left(a_{14}, \alpha_{d_{14}}, \alpha_{d_{24}}\right)\end{array}\right)$

First we compute,

$$
\begin{aligned}
& \operatorname{IWA}_{i}\left(a_{11}, \alpha_{d 11}, \alpha_{d 21}\right) \\
& \quad=\operatorname{IWA}\{\langle 0.4,0.5\rangle,\langle 0.4,0.6\rangle,\langle 0.5,0.4\rangle\} \\
& =\langle 0.444229,0.473037\rangle \\
& \operatorname{IWA}_{i}\left(a_{12}, \alpha_{d 12}, \alpha_{d 22}\right) \\
& =\operatorname{IWA}\{\langle 0.5,0.2\rangle,\langle 0.5,0.3\rangle,\langle 0.4,0.5\rangle\} \\
& =\langle 0.4602083,0.319995\rangle, \\
& \operatorname{IWA}_{i}\left(a_{13}, \alpha_{d 13}, \alpha_{d 23}\right) \\
& =\operatorname{IWA}\{\langle 0.7,0.2\rangle,\langle 0.5,0.4\rangle,\langle 0.5,0.3\rangle\} \\
& =\langle 0.586109,0.274286\rangle \\
& \operatorname{IWA}_{i}\left(a_{14}, \alpha_{d 14}, \alpha_{d 24}\right) \\
& =\operatorname{IWA}\{\langle 0.4,0.3\rangle,\langle 0.6,0.4\rangle,\langle 0.4,0.5\rangle\} \\
& =\langle 0.448974,0.39494\rangle .
\end{aligned}
$$

Then

$$
\begin{aligned}
& \delta_{1}= \mathrm{WG}^{\mathrm{GGIFSS}}(\langle 0.4,0.5\rangle,\langle 0.5,0.2\rangle, \\
&\langle 0.7,0.2\rangle\langle 0.4,0.3\rangle) \\
&=\operatorname{IWA}\left(\begin{array}{c}
(\langle 0.444229,0.473037\rangle, \\
\langle 0.4602083,0.319995\rangle, \\
\langle 0.586109,0.28744\rangle, \\
\langle 0.448974,0.39494\rangle)
\end{array}\right) \\
&=\langle 0.5185460,0.3764410\rangle
\end{aligned}
$$

Similarly, we can compute $\delta_{2}, \delta_{3}$ and $\delta_{4}$ and IFV are $\langle 0.514018,0.365981\rangle,\langle 0.4475176,0.441378\rangle,\langle 0.524296$, $0.277357\rangle$, respectively.

Now, by using score functions from Definition 2.4, $\delta_{1}$, $\delta_{2} \delta_{3}$ and $\delta_{4}$ are transmitted into real values $\eta\left(\delta_{1}\right)=$ $0.571052, \eta\left(\delta_{2}\right)=0.574018775, \eta\left(\delta_{3}\right)=0.5036698$ and $\eta\left(\delta_{4}\right)=0.62346925$, respectively. Thus $\xi_{4}$ is the most suitable alcohol-based HS on the assessments of both senior 
Table 5 GGIFSS $(\widehat{S}, \mathcal{B}, \widehat{g})$ in tabular form

\begin{tabular}{lll}
\hline$X \mid \mathcal{B}$ & $v_{1}$ & $v_{2}$ \\
\hline$\xi_{1}$ & $\langle 0.7,0.1\rangle$ & $\langle 0.6,0.2\rangle$ \\
$\xi_{2}$ & $\langle 0.6,0.3\rangle$ & $\langle 0.5,0.2\rangle$ \\
$\xi_{3}$ & $\langle 0.5,0.4\rangle$ & $\langle 0.4,0.4\rangle$ \\
\hline$\alpha_{d_{1}}$ & $\langle 0.5,0.2\rangle$ & $\langle 0.3,0.2\rangle$ \\
\hline
\end{tabular}

Table 6 GGIFSS $(\widehat{S}, \mathcal{B}, \widehat{g})$ in tabular form

\begin{tabular}{lll}
$X \mid \mathcal{B}$ & $v_{1}$ & $v_{2}$ \\
\hline$\xi_{1}$ & $\langle 0.4,0.3\rangle$ & $\langle 0.5,0.4\rangle$ \\
$\xi_{2}$ & $\langle 0.6,0.3\rangle$ & $\langle 0.5,0.5\rangle$ \\
$\xi_{3}$ & $\langle 0.5,0.4\rangle$ & $\langle 0.4,0.3\rangle$ \\
\hline$\alpha_{d_{1}}$ & $\langle 0.3,0.4\rangle$ & $\langle 0.3,0.4\rangle$ \\
\hline
\end{tabular}

experts as $\eta\left(\delta_{4}\right)=0.62346925$ is the maximum score. Thus choices of alcohol-based HSs are arrange in descending order $\xi_{4}>\xi_{2}>\xi_{1}>\xi_{3}$.

In case study 1 , extra inputs on IFSS are crucial assessments of senior person to finalize evaluation intake. Furthermore, the purpose of these essential additional inputs can run as customers demands in several scenarios. Therefore, we investigated a different case study in the next section.

\subsubsection{Case study 2}

Consider Example 3.3, where crucial extra inputs are the requirements for Mr. X and Mrs. X on attributes to buy a house. Accordingly, step 3 in the introduced algorithm can be viewed as the customers' demands in place of assessments on IFSS. We can follow other steps from the presented algorithm. So that GWA are calculated as follows: $\delta_{1}=$ $\langle 0.4599010,0.4069328\rangle, \delta_{2}=\langle 0.257387,0.505308\rangle, \delta_{3}=$ $\langle 0.241818,0.568510\rangle, \delta_{4}=\langle 0.290061,0.430839\rangle, \delta_{5}=$ $\langle 0.411085,0.000000\rangle$. Scores on GWA $\delta_{k}(k=1,2, \ldots, s)$ using Definition 2.4 are $\eta\left(\delta_{1}\right)=0.526484, \eta\left(\delta_{2}\right)=$ $0.376039, \eta\left(\delta_{3}\right)=0.336654, \eta\left(\delta_{4}\right)=0.429611, \eta\left(\delta_{5}\right)=$ 0.705542 , respectively. One can identify that $\xi_{5}$ is the acceptable house for both Mr. and Mrs. X. on their demands.

\section{Comparisons and discussion}

In this section, we analyze our method and outputs with already present methodologies.

\subsection{Comparisons}

In order to compare concepts of the proposed method, we compare our methodologies with recent major approaches by Feng et al. (2019) and Hayat et al. (2018b). For intended purpose, consider an example as follows:

Example 5.1 Consider GGIFSS as depicted in Table 5.

Let $\mathcal{B}=\left\{v_{1}, v_{2}\right\}$ be the set of attributes, $X=\left\{\xi_{1}, \xi_{2}, \xi_{3}\right\}$ is the set of alternatives, $\alpha_{d_{1}}$ is IFS for a moderator's assessments on IFSS in Table 5. Consider weighted vector $W=\left\{w_{0}, w_{1}\right\}=\{0.5,0.5\}$, where $w_{0}$ is weight over IFSS and $w_{1}$ is a weight over extra input. Intently, both weights in $W$ are equal, reason is that we have to analogous the weights with other methods for purpose of comparisons. Also, let $\left\{0.4 / v_{1}, 0.6 / v_{2}\right\}$ be a weighted vector over set of attributes. By using proposed aggregation operators, we have $\delta_{1}=0.6794, \delta_{2}=0.6270, \delta_{3}=0.5665$ and $\xi_{1}>\xi_{2}>\xi_{3}$. Thus $\xi_{1}$ is the best and optimal choice.

(1) Firstly, we examine our method with the framework presented by Hayat et al. (2018b). Let weighted vector $\left\{0.4 / v_{1}, 0.6 / v_{2}\right\}$ for attributes. By GGIFSS operators defined in Hayat et al. (2018b), we have $\ell_{1}=$ $0.4639, \ell_{2}=0.4112, \ell_{3}=0.3258$ and $\xi_{1}>\xi_{2}>\xi_{3}$. Thus $\xi_{1}$ is the best and optimal choice. This ranking is analogous to ranking of alternatives on proposed method.

(2) Feng et al. (2019) presented an aggregation operatorbased MCDM method. By using their method we obtain $Z_{J}\left(\xi_{1}\right)=0.7601, Z_{J}\left(\xi_{2}\right)=0.6539, Z_{J}\left(\xi_{3}\right)=0.5282$ and thus $\xi_{1}>\xi_{2}>\xi_{3}$. This ranking is analogous to ranking of alternatives on proposed method.

\subsection{Advantages of proposed method}

One of the prime advantages of new aggregation operators is assigning a weighted vector $\left\{w_{0}, w_{1}, \ldots, w_{p}\right\}$ on GGIFSS, where $w_{1}, w_{2}, \ldots, w_{p}$ are the weights on IFSs of extra inputs of $p$ number of moderators, respectively, and $w_{0}$ is a weight for IFSS-based data from a family of experts in GGIFSS. Certainly, this is a reality that preference or weights on IFSS and on extra input are reliable in several circumstances. As extra inputs are important opinions of senior experts or moderators for the final examination of evaluation (IFSS), thus in certain real-life situations their weights should be higher than $w_{0}$. On the other hand, in some real life situations weight $w_{0}$ on IFSS is also important, the fact that it shows a preference on data (or IFSS) provided by a family of experts on crucial assessments on alternatives. Manifestly, we consider an example to show the superiority of the proposed method:

Example 5.2 Consider GGIFSS as depicted in Table 6.

Where $\mathcal{B}=\left\{v_{1}, v_{2}\right\}$ is set of attributes with weighted vector $W=\left\{0.4 / v_{1}, 0.6 / v_{2}\right\}, X=\left\{\xi_{1}, \xi_{2}, \xi_{3}\right\}$ is the set of alternatives, $\alpha_{d_{1}}$ is IFS for moderator's assessments on IFSS in Table 6. By GGIFSS operators defined in Hayat et al. (2018b), we have $\ell_{1}=0.2644, \ell_{2}=0.2591, \ell_{3}=$ 0.2654 and $\xi_{3}>\xi_{1}>\xi_{2}$. It can be seen that $\alpha_{d_{1}}=$ $\{\langle 0.3,0.4\rangle,\langle 0.3,0.4\rangle\}$ and $\operatorname{IFWA}\{\langle 0.3,0.4\rangle,\langle 0.3,0.4\rangle\}=$ 
Table 7 GGIFSS $(\widehat{S}, \mathcal{B}, \widehat{g})$ in tabular form

\begin{tabular}{lll}
\hline$X \mid \mathcal{B}$ & $v_{1}$ & $v_{2}$ \\
\hline$\xi_{1}$ & $\langle 0.4,0.2\rangle$ & $\langle 0.4,0.2\rangle$ \\
$\xi_{2}$ & $\langle 0.6,0.4\rangle$ & $\langle 0.6,0.4\rangle$ \\
\hline$\alpha_{d_{1}}$ & $\langle 0.4,0.2\rangle$ & $\langle 0.6,0.4\rangle$
\end{tabular}

$\langle 0.3,0.4\rangle$. Here IFWA by Hayat et al. (2018b) examined again a same IFV $\langle 0.3,0.4\rangle$.

Consider weighted vector $W=\left\{w_{0}, w_{1}\right\}=\{0.3,0.7\}$, where $w_{0}$ is weight over IFSS and $w_{1}$ is weight over extra input. Notably, $w_{0}<w_{1}$ demonstrate that extra input/opinion has more weightage then IFSS from assessments of family of experts. Also, let $\left\{0.4 / v_{1}, 0.6 / v_{2}\right\}$ be a weighted vector over set of attributes. By using proposed aggregation operators, we have $\delta_{1}=0.4834, \delta_{2}=$ $0.4908, \delta_{3}=0.4831$ and $\xi_{2}>\xi_{1}>\xi_{3}$.

It concluded that if in an IFSs $\alpha_{d_{i}^{\prime}}$ (in GGIFSS) has same IFVs with respect each attributes then method of Hayat et al. (2018b), do not give precise aggregation with respect to attributes. Therefore, the purpose of moderator's input is diminished in such a scenario. On the other hand, this new method has advantage that it aggregates IFVs linearly with respect to attributes.

Feng et al. (2019), converted the extra opinion of moderator into score value or weightage in initial stages of decision making process.

Example 5.3 Consider GGIFSS as depicted in Table 7.

By the method of Feng et al. (2019), $\alpha_{d_{1}}$ is converted into scores vector as $\{0.6,0.6\}$ and further it converted to weighted vector $\{0.5,0.5\}$. It can be seen that alteration of $\alpha_{d_{1}}$ into weights finished the importance of memberships and non-memberships in IFVs. By using their method we obtain $Z_{J}\left(\xi_{1}\right)=0.6, Z_{J}\left(\xi_{2}\right)=0.6$, and thus it is difficult to choose the best alternative on the same value.

Now by proposed method we get $\delta_{1}=0.6132, \delta_{2}=$ 0.6125 and $\xi_{1}>\xi_{2}$. Thus alternatives can easily be ranked on proposed method as compared to method of Feng et al. (2019). Main advantage of our procedures is that it demonstrate perceptions or weights in a reasonable way for both IFSS and IFSs in GGIFSS.

From the above two examples, it is concluded that our method works better as compared to recent approaches Feng et al. (2019) and Hayat et al. (2018b). The method of Hayat et al. (2018) has disadvantages to tackling membership and non-membership, which are shown in Hayat et al. (2018b). On the other hand, inaccuracy of the models of the GIFSS (Agarwal et al. 2013) and GGIFSS (Garg and Arora 2018b) are shown in references (Feng et al. 2019; Khalil 2015; Hayat et al. 2018b), respectively. Hence we gave advantages and comparisons with recent prominent approaches. Consequently, the introduced results are better than recent methods of Feng et al. (2019) and Hayat et al. (2018b), and hence it is also better than others methods (Agarwal et al. 2013; Garg and Arora 2018b; Hayat et al. 2018).

The GGIFSS is a dimensionally very useful model for MCDM as it consists of components of initial judgment (as IFSS) and final judgments (as IFSs). Therefore, the proposed operators are well-defined to deal with each component of GGIFSS.

\section{Conclusions}

In this research, we have defined new aggregation operators (GWA) on GIFSS which are used to aggregate our work. Mainly, we have managed the weights with IFVs and their computation in a precise way. We have defined the preparatory work of decision making in an algorithm and studied its real-life application by dint of proposed methodology. Then we have presented the comparisons of our work with already existing methods and techniques comprising aggregation operators. The proposed foundation of aggregation instruments is principally possessing numerous advantages.

The way of computation of data from preferred results can sight wide applications in machine learning, applied intelligence, electrical engineering, supply chain managements, industrial designs, neural networks, and constructional engineering. This is especially because in such applications typically more experts/operators or data from different sources are available with different opinions/views. A proper aggregation of such views is thus beneficial and often necessary. Explicitly, we will define complexity analysis of algorithms to see the results in machine learning. The recognitions of a risk factor in management systems can be investigated on these key results.

Acknowledgements The third author acknowledges the Austrian Science Fund (FWF): Contract Number P32272-N38, acronym IL-EFS.

Author contributions Khizar Hayat developed the idea and wrote the paper. Khizar Hayat and Edwin Lughofer performed methodology. Khizar Hayat, Zalishta Tariq, and Edwin Lughofer were responsible for writing - original draft preparation. Khizar Hayat, M.Fahim Aslam and Edwin Lughofer reviewed and edited the paper.

\section{Declarations}

Conflict of interest The authors declare no conflict of interest.

Ethical approval The paper does not involve any research with human participants or animals involved by any of the authors. 


\section{References}

Acar U, Fatih K, Bekir T (2010) Soft sets and soft rings. Comput Math Appl 59(11):3458-3463

Agarwal M, Biswas K, Hanmandlu M (2013) Generalized intuitionistic fuzzy soft sets with applications in decision-making. Appl Soft Comput 13(8):3552-3566

Akram M, Shahzadi S (2018) Novel intuitionistic fuzzy soft multiple-attribute decision-making methods. Neural Comput Appl 29(7):435-447

Ali MI, Shabir M, Feng F (2017) Representation of graphs based on neighborhoods and soft sets. Int J Mach Learn Cyber 8(5):15251535

Atanassov K (1986) Intuitionistic fuzzy sets. Fuzzy Sets Syst 20(1):8796

Atanassov K (1999) Intuitionistic fuzzy sets: theory and applications. Physica-Verlag, Heidelberg/New York

Bashir M, Salleh AR, Alkhazaleh S (2012) Possibility intuitionistic fuzzy soft set. Adv Decis Sci 2012:1-11

Cagman N, Enginoglu S (2010) Soft set theory and uni-int decision making. Eur J Oper Res 207(2):848-855

Cagman N, Citak F, Enginoglu S (2010) Fuzzy parameterized fuzzy soft set theory and its applications. Turk J Fuzzy Syst 1(1):21-35

Chen D, Tsang E, Yeung D, Wang K (2005) The parameterization reduction of soft sets and its applications. Comput Math Appl 49(5-6):757-763

Das S, Kar S (2014) Group decision making in medical system: an intuitionistic fuzzy soft set approach. Appl Soft Comput 24:196211

Dengfeng L, Chuntian C (2001) New similarity measures of intutitionistic fuzzy sets and application to pattern recognitions. Pattern Recogn Lett 23(1-3):221-225

Deschrijver G, Kerre E (2003) On the relationship between some extensions of fuzzy set theory. Fuzzy Sets Syst 133(2):227-235

Fei L, Wang H, Chen L, Deng Y (2019) A new vector valued similarity measure for intuitionistic fuzzy sets based on owa operators. Iran J Fuzzy Syst 16(3):113-126

Feng F, Fujita H, Ali MI, Yager RR, Liu X (2019) Another view on generalized intuitionistic fuzzy soft sets and related multi-attribute decision making methods. IEEE Trans Fuzzy Syst 27(3):474-488

Garg H, Arora R (2018a) A nonlinear-programming methodology for multi-attribute decision-making problem with interval-valued intuitionistic fuzzy soft sets information. Appl Intell 48(8):20312046

Garg H, Arora R (2018b) Generalized and group-based generalized intuitionistic fuzzy soft sets with applications in decision-making. Appl Intell 48(2):343-356

Garg H, Kumar R (2018) An advanced study on the similarity measures of intuitionistic fuzzy sets based on the set pair analysis theory and their application in decision making. Soft Comput 22(15):4959 4970

Hayat K, Ali MI, Alcantud JCR, Cao BY, Tariq KU (2018a) Best concept selection in design process: an application of generalized intuitionistic fuzzy soft sets. J Intell Fuzzy Syst 35(5):5707-5720

Hayat K, Ali MI, Cao BY, Karaaslan F, Yang XP (2018b) Another view of aggregation operators on group-based generalized intuitionistic fuzzy soft sets: multiattribute decision making method. Symmetry 10(12):753

Hayat K, Ali MI, Karaaslan F, Cao BY, Shah MH (2020) Design concept evaluation using soft sets based on acceptable and satisfactory levels: an integrated topsis and shannon entropy. Soft Comput 24(3):2229-2263

Jing JLJ, PeiYi T, Bose RJ, McCarthy JR, Tharmalingam N, Madheswaran T (2020) Hand sanitizers: a review on formulation aspects, adverse effects, and regulations. Int J Environ Res Public Health 17(9):3326

Khalil AM (2015) Commentary on generalized intuitionistic fuzzy soft sets with applications in decision-making. Appl Soft Comput 37:519-520

Khan MJ, Kuman P, Liu P, Kuman W (2020a) An adjustable weighted soft discernibility matrix based on generalized picture fuzzy soft set and its applications in decision making. J Intell Fuzzy Syst 38(2):2103-2118

Khan MJ, Kuman P, Liu P, Kuman W (2020b) Another view on generalized interval valued intuitionistic fuzzy soft set and its applications in decision support system. J Intell Fuzzy Syst 4(Preprint):1-15

Liang Z, Shi P (2003) Similarity measure on intuitionistic fuzzy sets. Pattern Recogn Lett 24(15):2687-2693

Liu P, Chen SM, Wang Y (2020) Multiattribute group decision making based on intuitionistic fuzzy partitioned Maclaurin symmetric mean operators. Inf Sci 512:830-854

Maji PK, Biswas R, Roy AR (2001a) Fuzzy soft sets. J Fuzzy Math 9(3):589-602

Maji PK, Biswas R, Roy AR (2001b) Intuitionistic fuzzy soft sets. J Fuzzy Math 9(3):677-692

Maji PK, Biswas R, Roy AR (2003) Soft set theory. Comput Math Appl 45(4-5):555-562

Molodtsov D (1999) Soft set theory-first results. Comput Math Appl 37(4-5):19-31

Pei D, Miao D (2005) From soft sets to information systems. Proc IEEE Int Conf Granul Comput 2:617-621

Roy AR, Maji PK (2007) A fuzzy soft set theoretic approach to decision making problems. J Comput Appl Math 203(2):412-418

Selvachandran G, Maji P, Faisal R, Salleh A (2017) Distance and distance induced intuitionistic entropy of generalized intuitionistic fuzzy soft sets. Appl Intell 47(1):132-147

Shabir M, Naz M (2011) On soft topological spaces. Comput Math Appl 61(7):1786-1799

Szmidt E, Kacprzyk J (2000) Distances between intuitionistic fuzzy sets. Fuzzy Sets Syst 114(3):505-514

Tiwari V, Jain PK, Tandon P (2017) A bijective soft set theoretic approach for concept selection in design process. J Eng Des 28(2):100-117

Wang W, Xin X (2005) Distance measure between intuitionistic fuzzy sets. Pattern Recogn Lett 26(13):2063-2069

Xia MM, Xu ZS, Chen N (2013) Some hesitant fuzzy aggregation operators with their application in group decision making. Group Decis Negot 22(2):259-279

Xu Z (2007) Intuitionistic fuzzy aggregation operators. IEEE Trans Fuzzy Syst 15(6):1179-1187

Xu Z, Yager RR (2006) Some geometric aggregation operators based on intuitionistic fuzzy sets. Int J Gen Syst 35(4):417-433

Yin Y, Li H, Jun YB (2012) On algebraic structure of intuitionistic fuzzy soft sets. Comput Math Appl 64(9):2896-2911

Zadeh LA (1965) Fuzzy sets. Inf Control 8(2):338-353

Zimmermann HJ (2010) Fuzzy set theory. Wiley interdisciplinary reviews: computational statistics 2(3):317-332

Publisher's Note Springer Nature remains neutral with regard to jurisdictional claims in published maps and institutional affiliations. 